\title{
Modeling the Solubility of Dihydroxybenzoic Acid and Methylbenzoic Acid Isomers in Supercritical Carbon Dioxide
}

\author{
Authors: Loubna Nasri ${ }^{1 *}$, Salima Bensaad ${ }^{2}$, Zouhir Bensetiti ${ }^{3}$ \\ ${ }^{1}$ Department of Pharmaceutical Engineering, University Constantine 3, Algeria \\ ${ }^{2}$ Department of Chemistry, University Constantine 1, Algeria \\ ${ }^{3}$ Unisignal Inc., Brossard, Quebec, Canada \\ E-mail: ${ }^{1}$ loubnanasri@yahoo.com, ${ }^{2}$ salimabensaad@yahoo.com, ${ }^{3}$ zouhir.bensetiti@yahoo.com
}

\begin{abstract}
In this work, we propose to correlate and predict the solubility in supercritical $\mathrm{CO}_{2}$ of disubstituted aromatic isomers of hydroxybenzoic acid and methylbenzoic acid with a new methodology based on the expanded liquid theory, in which the solid-fluid equilibrium is modeled using the local composition model of UNIQUAC in which the interaction parameters are related to the solvent reduced density with an empiric exponential form equations. The experimental solubility of hydroxybenzoic acid isomers, methylbenzoic acid isomers and mixed isomers $(m-$ hydroxybenzoic acid $+p$-hydroxybenzoic acid) are used for evaluating the correlation and prediction capabilities of this new methodology. The results obtained using the proposed model show good agreement with the experimental data used.
\end{abstract}

\section{Keywords: Supercritical fluid extraction; aromatic isomers; solubility modeling; UNIQUAC.}

\section{Introduction}

During the past few years, widespread attention has been focused on supercritical fluids due to their potential application in extraction processes in food processing, pharmaceutical and petroleum industries, etc. The main advantages of supercritical fluid extraction over conventional extraction methods include increased speed, easy solvent separation and better recovery, and reduction in both solvent usage and solvent waste generation. The most widely used supercritical fluid is carbon dioxide because it is nontoxic, nonflammable and relatively inexpensive, and possesses reasonable critical properties $\left(T_{\mathrm{C}}=30{ }^{\circ} \mathrm{C}\right.$ and $P_{\mathrm{C}}=73$ bar) as well as a high solvent power for a wide range of nonpolar and intermediately polar organic compounds.

The solubility of a solute in a supercritical fluid is perhaps the most important thermo physical property that must be determined and modeled for an efficient design of any extraction procedure based on supercritical solvents. The determination of solubilities of a wide variety of solids and liquids of low volatility in supercritical carbon dioxide has received considerable attention in recent years. However, despite the vital importance of the solubility data of isomeric compounds from chemical, biochemical, pharmaceutical and industrial points of view, there is still a lack of fundamental solubility and mass-transfer data available in the literature to facilitate the development of commercial-scale processes. Since the experimental determination of the solubilities of various solutes in supercritical fluids at each operating condition is tedious, time-consuming and not reported in literatures, there is a considerable interest in mathematical models that can accurately predict the solubilities of solid solutes in supercritical fluids [1].
Aromatic isomers serve as raw materials for a wide variety of chemical, pharmaceutical, and polymer products; in some cases the individual isomers are difficult to obtain in pure form, and frequently the separation involves an ortho-para pair [2] because in most cases the solute-solute interactions lead to an enhancement in the solubilities of components relative to their respective binary systems [3]. As a consequence, there is now a greater need to understand the solubility behaviour of such systems. Therefore it is essential to have a model that not only can accurately correlate but also predict phase equilibrium properties.

Some of the models that have been used for correlating solubility data can be classified in two classes, equations of state based models (EOS) [4] and empirical models [5]. EOS based models require the prior knowledge of a certain number of parameters such as the critical properties (temperature and pressure), acentric factor and the sublimation pressure of the solid solute. These parameters are not available and are often calculated using group contribution methods, which could lead to solubility error prediction. Due to the lack of information on these properties, empirical models are used for the correlation of experimental solubility data. These models are known as density-based models and consist of equations that contain constants that are empirically adjusted for each compound. Although simple, these models rely much on the knowledge of the thermodynamic behaviour of the supercritical solvent rather than of the solute, and are mostly capable of correlating rather than predicting the solubility. They are used for quantitative determination of the solute solubility in supercritical phase at equilibrium, and do not provide qualitative information about the solute-solvent interaction.

In this work we will test the methodology previously used [6] that correlates and predicts the solubility of solids in supercritical carbon dioxide based on the expanded liquid 
model theory $[7,8]$. This theory does not require the knowledge of the solute critical properties and sublimation pressure.

In this case the supercritical phase is considered as an expanded liquid and is modeled using excess Gibbs energy models such as Margules, Van Laar, and local composition based models i.e. Wilson, NRTL and UNIQUAC. In this study we focus on the use of the UNIQUAC model that has been widely used in modeling vapour-liquid and liquidliquid equilibrium data. This model does not only take the size and nature of the molecules into consideration, but also accounts for the strength of solute-solvent intermolecular forces. And because the primary concentration variable is the surface fraction rather than mole fraction, the UNIQUAC model is applicable to solutions containing small or large molecules, including polymers.

\section{Model Development}

In the supercritical state, a fluid has a high density when compared with a gas. In fact, the density of a supercritical fluid is closer to that of a liquid than that of a gas. Consequently, in theoretical treatments the supercritical fluid phase can be treated approximately as an expanded liquid. This allows the phase equilibria between the solute and the supercritical fluid to be represented thermodynamically by solid-liquid equilibrium relations and conventional activity coefficients. To estimate the solid solubility in the supercritical phase, the knowledge of the activity coefficients are required. These coefficients are determined from the knowledge of the component fugacities, thus when the equilibrium of the solid and the supercritical phase is reached, we have:

$f_{2}^{\mathrm{s}}=f_{2}^{\mathrm{SCF}}=f_{2}^{\mathrm{L}}$

$f_{2}^{s}$ is the fugacity of the solute in the solid phase considered as pure solid and equal to $f_{2}^{o s}$ because the solubility of supercritical fluid (SCF) in the solid phase is considered to be negligibly small. $f_{2}^{L}$ is the fugacity of the solid solute in the supercritical phase and is equal to:

$f_{2}^{\mathrm{L}}=\gamma_{2} y_{2} f_{2}^{\mathrm{oL}}$

Eq. (1) could be written as follows:

$f_{2}^{\mathrm{os}}=\gamma_{2} y_{2} f_{2}^{\mathrm{oL}}$

where $\gamma_{2}, y_{2}$ and $f_{2}^{o L}$ are the activity coefficient, the solid solubility represented in mole fraction and the fugacity of the pure solid solute in the expanded liquid phase respectively. For the fugacity, according to Prausnitz et al. [9] we have:

$$
\begin{aligned}
& \ln \left(\frac{f_{2}^{\mathrm{os}}}{f_{2}^{\mathrm{ol}}}\right)= \\
& \frac{-\Delta H_{2}^{f}}{R}\left(\frac{1}{T}-\frac{1}{T_{m}}\right)-\frac{\Delta C_{p}}{R T}\left(\frac{T-T_{m}}{T}\right)+\frac{\Delta C_{p}}{R} \ln \left(\frac{T}{T_{m}}\right)
\end{aligned}
$$

The heat capacity terms can be neglected [9] and Eqs. (3) and (4) are then combined to yield an expression for the solute solubility: $y_{2}=\frac{1}{\gamma_{2}} \exp \left[\frac{-\Delta H_{2}^{f}}{R}\left(\frac{1}{T}-\frac{1}{T_{m}}\right)\right]$

where $\Delta H_{2}^{f}$ is the enthalpy of fusion, $T_{m}$ is the melting point temperature of the solid solute.

Since the solid solubility in the supercritical phase is very small, we can assume to be at infinite dilution condition. Consequently, the activity coefficient of the solid solute is the one at infinite dilution and the density of the solution is that of the pure solvent. Thus Eq. (5) becomes:

$y_{2}=\frac{1}{\gamma_{2}^{\infty}} \exp \left[\frac{-\Delta \mathrm{H}_{2}^{\mathrm{f}}}{\mathrm{R}}\left(\frac{1}{T}-\frac{1}{T_{m}}\right)\right]$

The activity coefficient of the solid solute at infinite dilution $\gamma_{2}^{\infty}$ was calculated using the UNIQUAC model which consists of two parts, a combinatorial part $\gamma_{2}^{C, \infty}$ that attempts to describe the dominant entropic contribution, and a residual part $\gamma_{2}^{R, \infty}$ that is due primarily to intermolecular forces that are responsible for the enthalpy of mixing. The combinatorial part is determined only by the composition and by the sizes and shapes of the molecules; it requires only pure-component data. The residual part, however, depends also on intermolecular forces; the two adjustable binary parameters $a_{12}$ and $a_{21}$, therefore, appear only in the residual part [9]:

$\ln \gamma_{2}^{\infty}=\ln \gamma_{2}^{\mathrm{C}, \infty}+\ln \gamma_{2}^{\mathrm{R}, \infty}$

$\ln \gamma_{2}^{\mathrm{C}, \infty}=1-\frac{r_{2}}{r_{1}}+\ln \frac{r_{2}}{r_{1}}-q_{2} \frac{z}{2}\left(1-\frac{r_{2} q_{1}}{r_{1} q_{2}}+\ln \frac{r_{2} q_{1}}{r_{1} q_{2}}\right)$

where $q$ and $r$ are the surface area and volume parameters; $z$ is the coordination number that is usually taken equal to 10 . The residual part at infinite dilution is given by the following equation [9]:

$\ln \gamma_{2}^{R, \infty}=q_{2}\left(1-\ln \tau_{12}-\tau_{21}\right)$

where

$\tau_{12}=\exp \left(-\Delta u_{12} / R T\right)=\exp \left(-a_{12} / T\right)$

$\tau_{21}=\exp \left(-\Delta u_{21} / R T\right)=\exp \left(-a_{21} / T\right)$

where $\Delta u_{12}$ and $\Delta u_{21}$ are characteristic energies and are related to the interaction parameters $\mathrm{a}_{12}$ and $\mathrm{a}_{21}$ through Eq. (10). Finally combining Eqs. (9) and (10) leads to

$\ln \gamma_{2}^{R, \infty}=q_{2} \frac{a_{12}}{T}+q_{2}\left(1-e^{\frac{-a_{21}}{T}}\right)$

Eq. (11) could be written in reduced form by introducing the reduced temperature, thus we obtain

$\ln \gamma_{2}^{R, \infty}=q_{2} \frac{a_{12}^{\prime}}{T r}+q_{2}\left(1-e^{\frac{-a_{21}^{\prime}}{T r}}\right)$

with $a_{12}^{\prime}=a_{12} / T_{c}$ and $a_{21}^{\prime}=a_{21} / T_{c}, T_{c}$ is the solvent critical temperature. 
The binary interaction parameters $a_{12}^{\prime}$ and $a_{21}^{\prime}$ are related to the energy of interaction between the solid solute and the solvent in the supercritical phase, and cannot be kept constant and especially at high pressure conditions. Therefore to take into account the pressure and temperature effects, these parameters are assumed to be density dependant and were fitted to the following equations:

$$
\begin{aligned}
& a_{12}^{\prime}=\alpha_{12} \cdot \exp \left(\beta_{12} \cdot \rho_{r}\right) \\
& a_{21}^{\prime}=\alpha_{21} \cdot \exp \left(\beta_{21} \cdot \rho_{r}\right)
\end{aligned}
$$

$\rho_{r}$ is the reduced density of the solvent equal to $\rho / \rho_{c}$ where $\rho_{c}$ is its critical density, $a_{12}, \beta_{12}, a_{21}$ and $\beta_{21}$ are the regressed parameters of the model.

\section{Isomers Solubility Calculation}

The surface area and volume parameters are calculated as the sum of the group volume and area parameters ( $R$ and Q) given by the UNIFAC group specifications [9]. Since the solutes are isomers they have the same functional groups so they have the same surface area and volume parameters: (hydroxybenzoic acid isomers: $r_{2}=4.6869$, $\mathrm{q}_{2}=3.624$ ) and (methylbenzoic acid isomers: $\mathrm{r}_{2}=5.058$, $\mathrm{q}_{2}=3.912$ ). These parameters and properties listed in Table 1 , together with those of carbon dioxide listed in Table 2 are used to calculate the combinatorial part of the activity coefficient from Eq. (8).

Table 1. Isomers Fusion Properties.

\begin{tabular}{lccc}
\hline Component & $T_{m}(\mathrm{~K})$ & $\Delta \mathrm{H}_{2}^{\mathrm{f}}(\mathrm{J} / \mathrm{mol})$ & $\begin{array}{c}\text { Solubility } \\
\text { Reference }\end{array}$ \\
\hline$m$-hydroxybenzoic acid & $476.0^{[12]}$ & $36500^{[12]}$ & {$[2],[3]$} \\
$p$-hydroxybenzoic acid & $488.1^{[12]}$ & $30990^{[12]}$ & {$[2],[15]$} \\
$o$-hydroxybenzoic acid & $432.0^{[13]}$ & $19585^{[13]}$ & {$[2],[3]$,} \\
& & & {$[15],[16]$} \\
$m$-methylbenzoic acid & $381.9^{[14]}$ & $15730^{[14]}$ & {$[17]$} \\
$p$-methylbenzoic acid & $452.8^{[14]}$ & $22720^{[14]}$ & {$[17]$} \\
$o$-methylbenzoic acid & $376.9^{[14]}$ & $20170^{[14]}$ & {$[17]$} \\
\hline
\end{tabular}

In other hand, Eq. (12) is used to calculate the residual part of the solid solute activity coefficient. Thermodynamic properties of the solid solute listed in Table 1 are used together with Eqs. (7), (8), and (12) to estimate the solubility $y_{2}$ using Eq. (6). The interaction parameters $a_{12}^{\prime}$ and $a_{21}^{\prime}$ are then regressed according to Eqs. (13a) and (13b) using the solver tool in Excel [10]. The best regression is based on minimizing the error between the regressed and experimental solubility data. The definition of the error is based on the work of Valderama et al. [11] and the objective function that minimizes the sum of average absolute relative deviation (AARD) is

$$
\operatorname{AARD}(\%)=\frac{100}{n p} \sum_{1}^{n p} \frac{\left|y_{2(\exp )}-y_{2(c a l)}\right|}{y_{2(\exp )}}
$$

where $n p$ is the experimental number of points, and $y_{2(\exp )}$ and $y_{2 \text { (cal) }}$ are the solubility of isomer obtained from experimental data and calculated by thermodynamic model, respectively.
Table 2. Solvent Physical Properties.

\begin{tabular}{ccccccc} 
Solvent & $T_{c}(\mathrm{~K})$ & $P_{c}$ (bar) & $\begin{array}{c}\rho_{c}\left(\mathrm{~mol} / \mathrm{cm}^{3}\right) \\
\text { x } 100\end{array}$ & $r_{1}$ & $q_{1}$ & Ref. \\
\hline $\mathrm{CO}_{2}$ & 304.2 & 73.83 & 1.063 & 1.296 & 1.261 & {$[9],[18]$}
\end{tabular}

\section{Correlation Results}

The interaction parameters $a_{12}$ and $a_{21}$ are regressed through the optimization of the adjustable parameters $a_{12}$, $\beta_{12}, a_{21}$ and $\beta_{21}$. These fitting parameters are evaluated by minimizing the objective function given in Eq. (14).

The analysis of the model results is done through statistical calculations. Tables 3 and 4 provide the quantitative results of the regression for the proposed model. The AARD is listed for each isomer and for each temperature together with the adjustable parameters values and the overall absolute deviations.

\subsection{Results and Discussions}

Each isomer parameters are obtained by fitting its own and whole solubility data. The overall deviations values obtained are generally low and so indicate a good correlation capability of the model.

From Table 3 and Figure 2 we can see clearly that for the hydroxybenzoic acid isomers, the greatest values of AARD are noted at high temperatures especially for $\mathrm{T}=373 \mathrm{~K}$. These can be probably attributed to the melting point depression occurring in some high-pressure-mixtures $[19,20]$.

\begin{tabular}{|c|c|c|c|c|c|c|c|}
\hline Comp. & $n p$ & $T(\mathrm{~K})$ & $\alpha_{12}$ & $\beta_{12}$ & $\alpha_{21}$ & $\beta_{21}$ & $\begin{array}{c}\text { AARD } \\
(\%)\end{array}$ \\
\hline \multirow[t]{4}{*}{$m-$} & 16 & 318 & 4.24 & -0.292 & 6497.8 & -14.5 & 1.91 \\
\hline & & 328 & & & & & 8.23 \\
\hline & & 373 & & & & & 30.1 \\
\hline & & overall & & & & & 11.32 \\
\hline \multirow[t]{6}{*}{$o-$} & 84 & 308 & 2.83 & -0.288 & 2.35 & -2.2 & 8.60 \\
\hline & & 313 & & & & & 7.48 \\
\hline & & 318 & & & & & 11.04 \\
\hline & & 328 & & & & & 5.74 \\
\hline & & 373 & & & & & 41.95 \\
\hline & & overall & & & & & 10.37 \\
\hline \multirow[t]{4}{*}{$p-$} & 16 & 318 & 4.57 & -0.292 & 6100.3 & -14.9 & 2.28 \\
\hline & & 328 & & & & & 6.35 \\
\hline & & 373 & & & & & 30.40 \\
\hline & & overall & & & & & 10.84 \\
\hline
\end{tabular}

Table 3. Regression Results for Hydroxybenzoic Acid Isomers.

Under the influence of high-pressure carbon dioxide, organic solids may undergo melting point depression [21] which lead to the exhibition of fluid-liquid equilibria and so affect the measured solubility data. Lucien \& Foster [15] have mentioned that with their experimental technique for measuring, in all of the systems investigated (pure and mixed) no melting point depression was observed. However, Krukonis \& Kurnik [2] have reported measured 
solubilities of the hydroxybenzoic acid isomers at very high conditions ( $T=373 \mathrm{~K}$ and $P>207$ bar) without indication to the melting point depression phenomenon.

Table 4. Regression Results for Methylbenzoic Acid Isomers.

\begin{tabular}{|c|c|c|c|c|c|c|c|}
\hline Comp. & $\mathrm{np}$ & $\mathrm{T}(\mathrm{K})$ & $\alpha_{12}$ & $\beta_{12}$ & $\alpha_{21}$ & $\beta_{21}$ & $\begin{array}{c}\text { AARD } \\
(\%)\end{array}$ \\
\hline \multirow[t]{4}{*}{$m-$} & 18 & 313 & 1.8 & -0.112 & 11.2 & -2.6 & 8.27 \\
\hline & & 323 & & & & & 8.50 \\
\hline & & 333 & & & & & 16.20 \\
\hline & & overall & & & & & 11.01 \\
\hline \multirow[t]{4}{*}{$o-$} & 18 & 313 & 1.62 & -0.086 & 10.49 & -2.41 & 9.50 \\
\hline & & 323 & & & & & 5.60 \\
\hline & & 333 & & & & & 16.30 \\
\hline & & overall & & & & & 10.05 \\
\hline \multirow[t]{4}{*}{$p-$} & 18 & 313 & 2.09 & -0.174 & 4.08 & -1.85 & 2.20 \\
\hline & & 323 & & & & & 2.70 \\
\hline & & 333 & & & & & 5.80 \\
\hline & & overall & & & & & 3.60 \\
\hline
\end{tabular}

\section{Predictions}

A number of aromatic isomers serve as raw materials for a wide variety of chemical, pharmaceutical, and polymer products and in most cases the individual isomers are difficult to obtain in pure form because binary solubility data present a limited picture of the complex interactions that can occur in the supercritical fluid phase [2]. Consequently, the separation involves a mixture of isomers and frequently an ortho-para pair. In this case of mixture solute-solute interactions lead to an enhancement in the solubility of components relative to their respective binary systems [4]. As a consequence, in literature there are several studies on the determination of solubilities of mixed isomers in supercritical carbon dioxide.

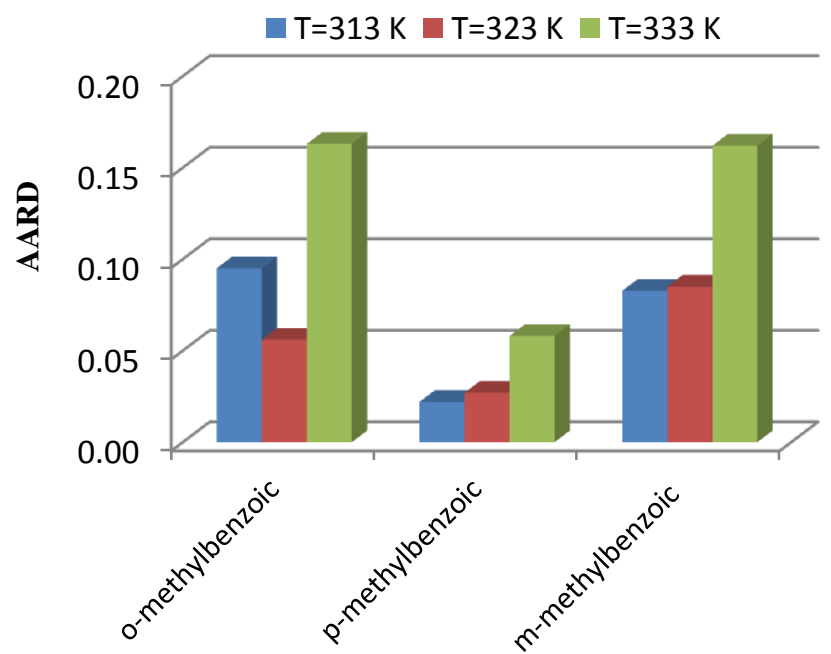

Figure 1. Comparison of the (AARD) for the methylbenzoic acid isomers.

In this part, we attempt to predict the solubilities of mixed hydroxybenzoic acid isomers in supercritical carbon dioxide. Experimental solubility data provided by Lucien \&
Foster [15] for an equimolar mixture of the isomers $m$ hydroxybenzoic acid and $p$-hydroxybenzoic acid are used in this case. The solubility data of the two isomers are very small and have an order of $10^{-6}$. As a consequence, we can assume that the density of the supercritical phase is that of the pure solvent, and the activity coefficient of each isomer is the one at infinite dilution. In this case the only interaction parameters that are taken into account are those of $m$-hydroxybenzoic- $\mathrm{CO}_{2}$ and $p$-hydroxybenzoic- $\mathrm{CO}_{2}$. Therefore the solubilities are estimated using Eqs. (6) to (13b) and interaction parameters for both meta and para isomers listed in Table 3 are directly implemented to estimate the solubility of each isomer in the mixture.

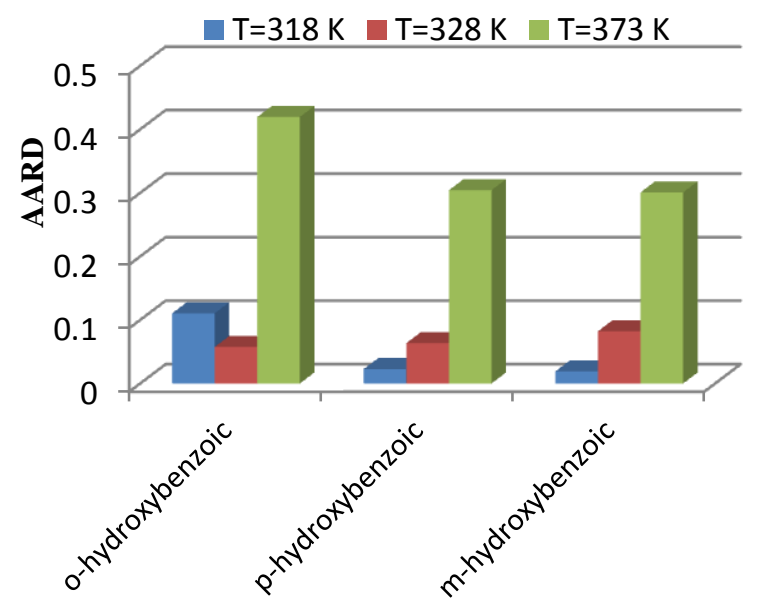

Figure 2. Comparison of the (AARD) for the hydroxybenzoic acid isomers.

The absolute average relative deviations (AARD) obtained are given in Table 5. Figures 3 and 4 shows a parity plot of the experimental versus predicted solubility data of mixed $m$-hydroxybenzoic and $p$-hydroxybenzoic isomers in supercritical $\mathrm{CO}_{2}$ for two different temperatures, i.e. 318 and $328 \mathrm{~K}$.

Table 5. Results of Predictions.

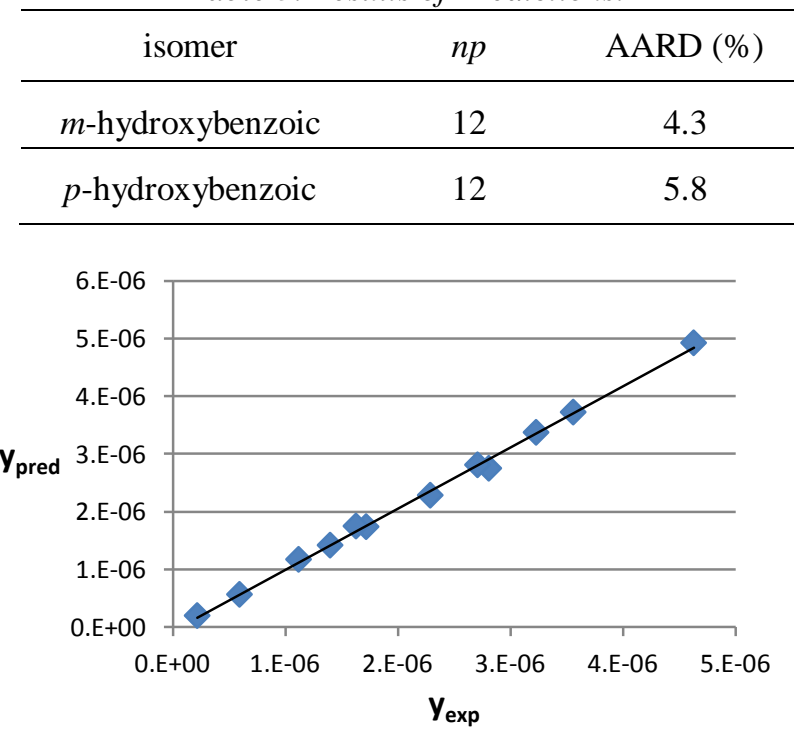

Figure 3. Comparison of predicted with experimental solubility of m-hydroxybenzoic acid at $T=318 \mathrm{~K}$ and $T=328 K$.

Figure 3, Figure 4, and results in Table 5 show good agreement between measured solubility data and predicted 
ones and confirm predictive ability of the proposed model.

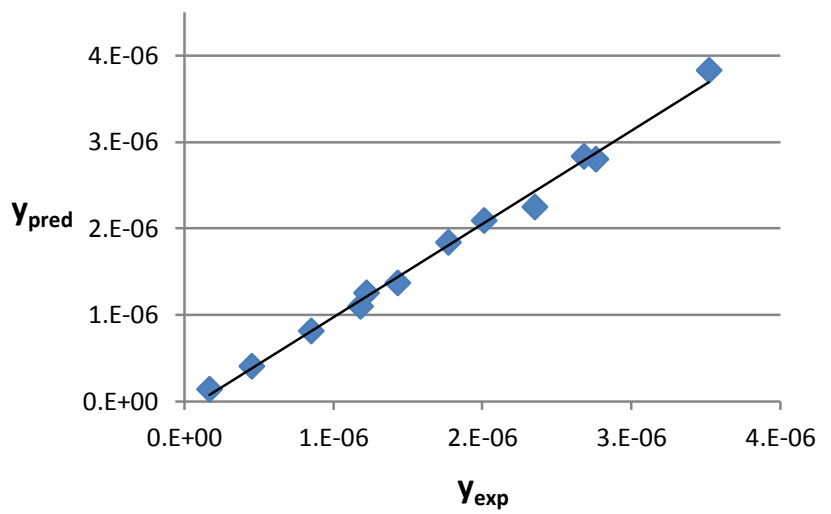

Figure 4. Comparison of predicted with experimental solubility of p-hydroxybenzoic acid at $T=318 \mathrm{~K}$ and $T=328 K$

\section{Conclusions}

In this work, we have proposed the correlation and prediction of the solubility in supercritical $\mathrm{CO}_{2}$ of disubstituted aromatic isomers of hydroxybenzoic acid and methylbenzoic acid with a new methodology based on the expanded liquid theory, in which the solid-fluid equilibrium is modeled using the local composition model of UNIQUAC. The results obtained using the proposed model show good agreement with the experimental data of isomers used. Moreover predictive capabilities of the proposed model for solid solubility were well demonstrated for mixed isomers-solvent systems.

\section{References}

[1] G. Madras, C. Kulkarni, J. Modak, Modeling the solubilities of fatty acids in supercritical carbon dioxide, Fluid Phase Equilibria, 209, 207-213, 2003.

[2] Val J. Krukonis, R T. Kurnik, Solubility of solid aromatic isomers in carbon dioxide, Journal of Chemical Engineering Data, 30, 247-249, 1985.

[3] F. P. Lucien, N. R. Foster, Solubilities of mixed hydroxybenzoic acid isomers in supercritical carbon dioxide, J. Chemical Engineering Data, 43, 726-731, 1998.

[4] N. R. Foster, G. S. Gurdial, J. S. L. Yun, K. K. Liong, K. D. Tilly, S. S. T. Ting, H. Singh, J. H. Lee, Significance of the crossover pressure in solidsupercritical fluid phase equilibria, Ind. Eng. Chem. Res, 30, 1955-1964, 1991.

[5] J. Chrastil, Solubility of solids and liquids in supercritical gases, Journal of Physical Chemistry, 86, 3016-3021, 1982.

[6] L. Nasri, Z. Bensetiti, S. Bensaad, Correlation of the solubility of some organic aromatic pollutants in supercritical carbon dioxide based on the UNIQUAC equation, Energy Procedia, 18, 1261-1270, 2012.

[7] J. W. Lee, J. M. Min, H. K. Bae, Solubility measurement of disperse dyes in supercritical carbon dioxide, Journal of Chemical Engineering Data, 44, 684-687, 1999.

[8] J. W. Lee, M. W. Park, H. K. Bae, Measurement and correlation of dye solubility in supercritical carbon dioxide, Fluid Phase Equilibria, 173, 277-284, 2000.

[9] J. M. Prausnitz, R. N. Lichtenthaler, E. G. De Azevedo, Molecular Thermodynamics of Fluid-Phase Equilibria, 3rd Ed. Prentice Hall Inc, 1999.

[10] D. L. Sparks, R. Hernandez and A. Estevez, Evaluation of density-based models for the solubility of solids in supercritical carbon dioxide and formulation of a new model, Chemical Engineering Science, 63, 42924301, 2008.

[11] J. O. Valderama, V. H. Alvarez, Correct way of reporting results when modelling supercritical phase equilibria using equations of state, The Canadian Journal of Chemical Engineering, 83, 578-581, 2005.

[12] S. S. Pinto, P. Diogo Hermı'nio, Energetics of hydroxybenzoic acids and of the corresponding carboxyphenoxyl Radicals, J. Phys. Chem. A, 109, 9700-9708, 2005 .

[13] S. E. Guigard, W. H. Stiver, A density-dependant solute solubility parameter for correlating solubilities in supercritical fluids, Ind. Eng. Chem. Res, 37, 37863792, 1998.

[14] NIST database (accessed 2012, Dec. 10) [Online] Available: http://webbook.nist.gov/chemistry/nameser.html.

[15] F. P. Lucien, N. R. Foster, Influence of matrix composition on the solubility of hydroxybenzoic acid isomers in supercritical carbon dioxide, Ind. Eng. Chem. Res, 35, 4686-4699, 1996.

[16] J. Ke, C. Mao, M. Zhong, B. Han, and H. Yan, Solubilities of salicylic acid in SC Co2 with ethanol co solvent, Journal of Supercritical Fluids, 9, 82-87, 1996.

[17] K. L. Tsai, F. N. Tsai, Solubilities of methylbenzoic acid isomers in supercritical carbon dioxide, Journal of Chemical Engineering Data, 40, 264-266, 1995.

[18] M. V. da Silva, D. Barbosa, Prediction of the solubility of aromatic components of wine in carbon dioxide, Journal of Supercritical Fluids, 31, 9-25, 2004.

[19] T. Bamberger, J. C. Erickson, C. L. Cooney, S. K. Kumar, Measurement and model prediction of solubilities of pure fatty acids, pure triglycerides, and mixtures of triglycerides in supercritical carbon dioxide, J. Chem. Eng. Data, 33, 327-333, 1988.

[20] H. Chang, D. G. Morrell, Solubilities of methoxy-1tetralone and methyl nitrobenzoate isomers and their mixtures in supercritical carbon dioxide, J. Chem. Eng. Data, 30, 74-78, 1985.

[21] S T. Chung, K. S. Shing, Multiphase behavior of binary and ternary systems of heavy aromatic hydrocarbons with supercritical carbon dioxide, Fluid Phase Equilibria, 81, 321-341, 1992. 